\title{
Does the accessory pancreatic duct have any protective role in acute biliary pancreatitis? Anatomical findings from cadaver dissections
}

\section{Introduction}

Acute pancreatitis is one of the most frequent gastrointestinal diseases for hospital admission in Latin American. It is represented by an inflammatory process of pancreas with multiple etiological agents. In our country, acute pancreatitis associated with gallbladder disease is generally considered to be due to obstruction of de pancreatic outflow that determine a high ductal pancreatic pressure or bile reflux as a enzymatic trigger mechanism. Although its physiopathology is complex, the answer to their understanding knowledge is based on the anatomy of pancreas. However the cognizance of the protective factors of pancreatitis is most important to research. One more time, the pancreatic anatomy is the key. The accessory pancreatic duct, first described by Santorini in 1775, is the smaller and less constant pancreatic duct, but is the main drainage duct of the dorsal pancreatic bud in the embryo. ${ }^{1}$ A patent of accessory pancreatic duct might protect the pancreas from the harmful consequences of obstruction of the Wirsung duct, but also promote biliary pancreatic reflux during obstruction of the common channel. ${ }^{2}$ Our aim is to determine the anatomy and morphology of this duct and its variations.

\section{Methods}

The anatomic study was conducted on 50 human cadavers after their fixation in formaldehyde $5 \%$, the duodenal-pancreatic blocks were dissected with microsurgical instruments and optical magnification. The cadaveric materials were used under the approval of the Department of Bioethics of the Institution based on the moral principle of human respect. Patency was evaluated with contrast and dye injection and results were documented with digital photography.

\section{Results}

Accessory pancreatic duct was present in $60 \%$ of the cadavers; the length ranged between 9 and $65 \mathrm{~mm}$, with a mean of $29.48 \mathrm{~mm}$ and the width, between 1 and $4 \mathrm{~mm}$ with a mean of $1.9 \mathrm{~mm}$. In 15 of the specimens Santorini's duct showed patency and in 20, an affluent from the uncinate process was found. The following types may be considered relating patency (A: patent, B: not patent) and diameter (1: $<2 \mathrm{~mm}, 2: \geq 2 \mathrm{~mm}$ ): A1,5;A2, 10;B1,7 and B2,8 (Graph 1\& Graph 2).

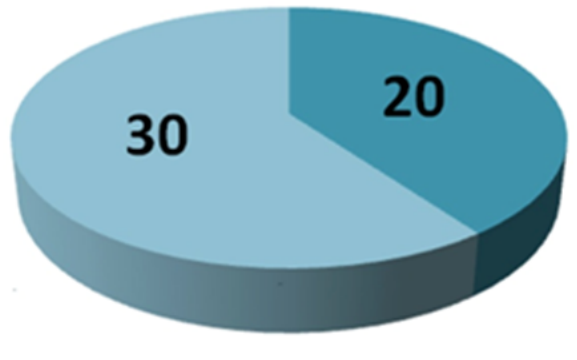

Graph I Prevalence of accessory pancreatic duct.
Volume 2 Issue 7 - 2016

\section{Lucas Nahuel Pina}

Department of Anatomy, Hospital de Clinicas José de San Martin, University of Buenos Aires, Argentina

Correspondence: Lucas Nahuel Pina, Department of Anatomy, Hospital de Clinicas José de San Martin, University of Buenos Aires, Argentina, Tel +54II50292489,

Email lucasnpina@gmail.com

Received: August 04, 2016| Published: November 09, 2016

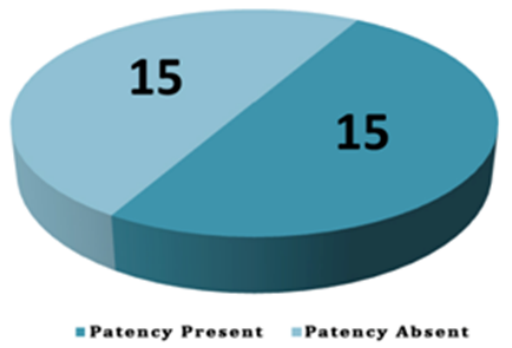

Graph 2 Accessory pancreatic duct patency rates.

\section{Conclusion}

The anatomical variations of the accessory pancreatic duct may have clinical importance; especially the type A2 which may protect the pancreas from the effects of Wirsung duct's obstruction during an episode of biliary acute pancreatitis. ${ }^{3}$ The patency of the accessory pancreatic duct could have a protective role in acute pancreatitis.

\section{Acknowledgements}

None.

\section{Conflict of interest}

Author declares that there is no conflict of interest.

\section{References}

1. Kamisawa T. Clinical significance of the minor duodenal papilla and accessory pancreatic duct. J Gastroenterol. 2004;39(7):605-615.

2. Arendt T, Stoffregen C, Kloehn S, et al. Santorini's duct--risk factor for acute pancreatitis or protective morphologic variant? Experiments in rabbits. Eur J Gastroenterol Hepatol. 1997;9(6):569-573.

3. Van Geenen EJ, van der Peet DL, Bhagirath P, et al. Etiology and diagnosis of acute biliary pancreatitis. Nat Rev Gastroenterol Hepatol. 2010;7(9):495-502. 